\title{
BMJ Open Electrophysiological ventricular substrate of stroke: a prospective cohort study in the Atherosclerosis Risk in Communities (ARIC) study
}

\author{
John A Johnson, ${ }^{1}$ Kazi T Haq, ${ }^{1}$ Katherine J Lutz, ${ }^{1}$ Kyle K Peters, ${ }^{1}$ \\ Kevin A Paternostro, ${ }^{1}$ Natalie E Craig, ${ }^{1}$ Nathan W L Stencel, ${ }^{1}$ Lila F Hawkinson, ${ }^{1}$ \\ Maedeh Khayyat-Kholghi, ${ }^{1}$ Larisa G Tereshchenko (D) ${ }^{1,2}$
}

To cite: Johnson JA, Haq KT, Lutz KJ, et al. Electrophysiological ventricular substrate of stroke: a prospective cohort study in the Atherosclerosis Risk in Communities (ARIC) study. BMJ Open 2021;11:e048542. doi:10.1136/ bmjopen-2020-048542

- Prepublication history and additional supplemental material for this paper are available online. To view these files, please visit the journal online (http://dx.doi.org/10.1136/ bmjopen-2020-048542).

Received 30 December 2020 Accepted 16 August 2021

Check for updates

(c) Author(s) (or their employer(s)) 2021. Re-use permitted under CC BY-NC. No commercial re-use. See rights and permissions. Published by BMJ.

${ }^{1}$ Department of Medicine, Cardiovascular Division or Knight Cardiovascular Institute, School of Medicine, Oregon Health \& Science University, Portland, Oregon, USA

${ }^{2}$ Department of Medicine, Johns Hopkins School of Medicine, Baltimore, Maryland, USA

Correspondence to Dr Larisa G Tereshchenko; tereshch@ohsu.edu

\section{ABSTRACT}

Objectives The goal of the study was to determine an association of cardiac ventricular substrate with thrombotic stroke (TS), cardioembolic stroke (ES) and intracerebral haemorrhage (ICH).

Design Prospective cohort study.

Setting The Atherosclerosis Risk in Communities (ARIC) study in 1987-1989 enrolled adults (45-64 years), selected as a probability sample from four US communities (Minneapolis, Minnesota; Washington, Maryland; Forsyth, North Carolina; Jackson, Mississippi). Visit 2 was in 1990 1992, visit 3 in 1993-1995, visit 4 in 1996-1998 and visit 5 in 2011-2013.

Participants ARIC participants with analysable ECGs and no history of stroke were included $(n=14479$; age $54 \pm 6$ y; $55 \%$ female; $24 \%$ black). Ventricular substrate was characterised by cardiac memory, spatial QRS-T angle (QRS-Ta), sum absolute QRST integral (SAIQRST), spatial ventricular gradient magnitude (SVGmag), premature ventricular contractions (PVCs) and tachycardia-dependent intermittent bundle branch block (TD-IBBB) on 12-lead ECG at visits 1-5.

Outcome Adjudicated TS included a first definite or probable thrombotic cerebral infarction, ES - a first definite or probable non-carotid cardioembolic brain infarction. Definite ICH was included if it was the only stroke event.

Results Over a median 24.5 years follow-up, there were 899 TS, 400 ES and 120 ICH events. Cox proportional hazard risk models were adjusted for demographics, cardiovascular disease, risk factors, atrial fibrillation, atrial substrate and left ventricular hypertrophy. After adjustment, PVCs (HR 1.72; 95\% $\mathrm{Cl} 1.02$ to 2.92), QRSTa (HR 1.15; 95\% Cl 1.03 to 1.28), SAIQRST (HR 1.20; $95 \% \mathrm{Cl} 1.07$ to 1.34) and time-updated SVGmag (HR 1.19; $95 \% \mathrm{Cl} 1.08$ to 1.32 ) associated with ES. Similarly, PVCs (HR 1.53; 95\% Cl 1.03 to 2.26), QRS-Ta (HR 1.08; 95\% Cl 1.01 to 1.16 ), SAIQRST (HR $1.07 ; 95 \% \mathrm{Cl} 1.01$ to 1.14 ) and time-updated SVGmag (HR 1.11; $95 \% \mathrm{Cl} 1.04$ to 1.19) associated with TS. TD-IBBB (HR 3.28; $95 \% \mathrm{Cl} 1.03$ to 10.46) and time-updated SVGmag (HR 1.23; 95\% Cl 1.03 to 1.47) were associated with ICH.

Conclusions PVC burden (reflected by cardiac memory) is associated with ischaemic stroke. Transient cardiac memory (likely through TD-IBBB) precedes ICH.

\section{STRENGTHS AND LIMITATIONS OF THIS STUDY}

$\Rightarrow$ This large prospective cohort with long-term followup and well-adjudicated stroke events provided sufficient statistical power for rigorous adjustment.

$\Rightarrow$ While the statistical power for ischaemic stroke was adequate, there were fewer intracerebral haemorrhage events, which challenged a fair comparison of models for ischaemic and haemorrhagic stroke events.

$\Rightarrow$ Thrombotic stroke (TS) included non-embolic and arterial embolic stroke (ES). The combined analysis of these two subtypes of ischaemic stroke is suboptimal and did not discriminate between these two types of TS.

$\Rightarrow$ In 2017, the American College of Cardiology/ American Heart Association guideline lowered the threshold for the definition of hypertension in adults $(\geq 130 / 80 \mathrm{~mm} \mathrm{Hg})$. We kept the historical definition of hypertension $(\geq 140 / 90 \mathrm{~mm} \mathrm{Hg}$ ) and also adjusted for actual blood pressure values, considering the paramount importance of hypertension as a stroke risk factor

$\Rightarrow$ Over the study period, the precision of stroke diagnosis has been strengthened, and cerebral amyloid angiopathy diagnostic criteria have been changed.

$\Rightarrow$ Assessment of paroxysmal arrhythmic events (atrial fibrillation (AF), premature atrial complexes, premature ventricular contractions (PVCs), tachycardiadependent intermittent bundle branch block) on a 10 s ECG cannot accurately represent the burden of arrhythmia. It is possible that the analyses were not sufficiently adjusted for AF, and the association between PVC and ES is weaker than reported.

\section{INTRODUCTION}

Stroke remains the leading cause of longterm disability and the fifth-leading cause of death in the USA. ${ }^{1}$ Despite declining stroke incidence in adults over 65 years of age, ${ }^{2}$ the global lifetime risk of stroke increased from $22.8 \%$ in 1990 to $24.9 \%$ in $2016 .^{3}$ Large portions, approximately $90.5 \%$ of 
stroke risk, could be attributed to traditional cardiovascular risk factors, such as hypertension, obesity, hyperlipidaemia, hyperglycaemic, renal dysfunction, smoking and sedentary lifestyle, as well as air pollution. ${ }^{4}$ Nevertheless, a recent genome-wide association study of stroke identified 22 previously unknown genetic loci, pointing towards additional, currently unrecognised mechanisms of stroke. ${ }^{5}$ The most accurate stroke risk prediction is provided by $\mathrm{CHA}_{2} \mathrm{DS}_{2}-\mathrm{VASc}$ and $\mathrm{P}_{2}-\mathrm{CHA}_{2} \mathrm{DS}_{2}$ VASc risk scores $^{6}$ in persons with and without ${ }^{7}$ atrial fibrillation $(\mathrm{AF})$. However, C-statistics for the stroke risk scores remain suboptimal ( 0.6 to 0.7). Thus, it is essential to understand additional factors beyond those already accounted for in the risk assessment models.

Several studies reported an association of both premature ventricular complexes (PVCs) and left ventricular hypertrophy $(\mathrm{LVH})$ with incident stroke. ${ }^{8-11}$ This suggests that an abnormal ventricular substrate, with or without atrial myopathy, may serve as an independent risk factor. Furthermore, it was previously shown that left ventricular interstitial fibrosis is associated with interatrial conduction abnormalities, characterised by $\mathrm{P}$-wave indices. ${ }^{12}$ However, the role of a ventricular substrate in stroke mechanisms is not entirely clear.

Global electrical heterogeneity (GEH) recently emerged as a global measure of an abnormal electrophysiological substrate in the ventricles of the heart. ${ }^{13} \mathrm{GEH}$ is a concept based on a spatial ventricular gradient (SVG) theory. ${ }^{14}$ It was mathematically demonstrated that the sum of the QRS and T vectors should theoretically be zero if depolarisation and repolarisation occurred in the same manner. By measuring the SVG, one could quantify the global dispersion of activation and recovery. SVG is independent of the type of ventricular activation..$^{1516}$ Therefore, it can be measured in individuals with or without $\mathrm{AF}$ or ventricular conduction abnormalities, facilitating a study of ventricular substrate. An association of GEH with stroke has not been previously studied.

We conducted this study with the goal of uncovering novel risk factors of stroke, to determine an association of electrophysiological ventricular substrate (quantified by GEH and traditional ECG metrics) with thrombotic, cardioembolic or haemorrhagic stroke subtypes. We hypothesised that GEH is associated with incident stroke in the Atherosclerosis Risk in Communities (ARIC) study participants.

\section{METHODS}

\section{Study population}

The ARIC study enrolled 15792 participants (age 45-64 years) between 1987 and $1989 .{ }^{17}$ In the current study, we included ARIC study participants with recorded resting 12-lead ECG and measured GEH ${ }^{13} ; n=15776$. ECGs were recorded during visits $1-5$. We excluded participants with a prevalent stroke (defined ${ }^{18}$ as previous stroke or transient ischaemic attack identified by a standardised interview) that occurred before the first ECG GEH measurement

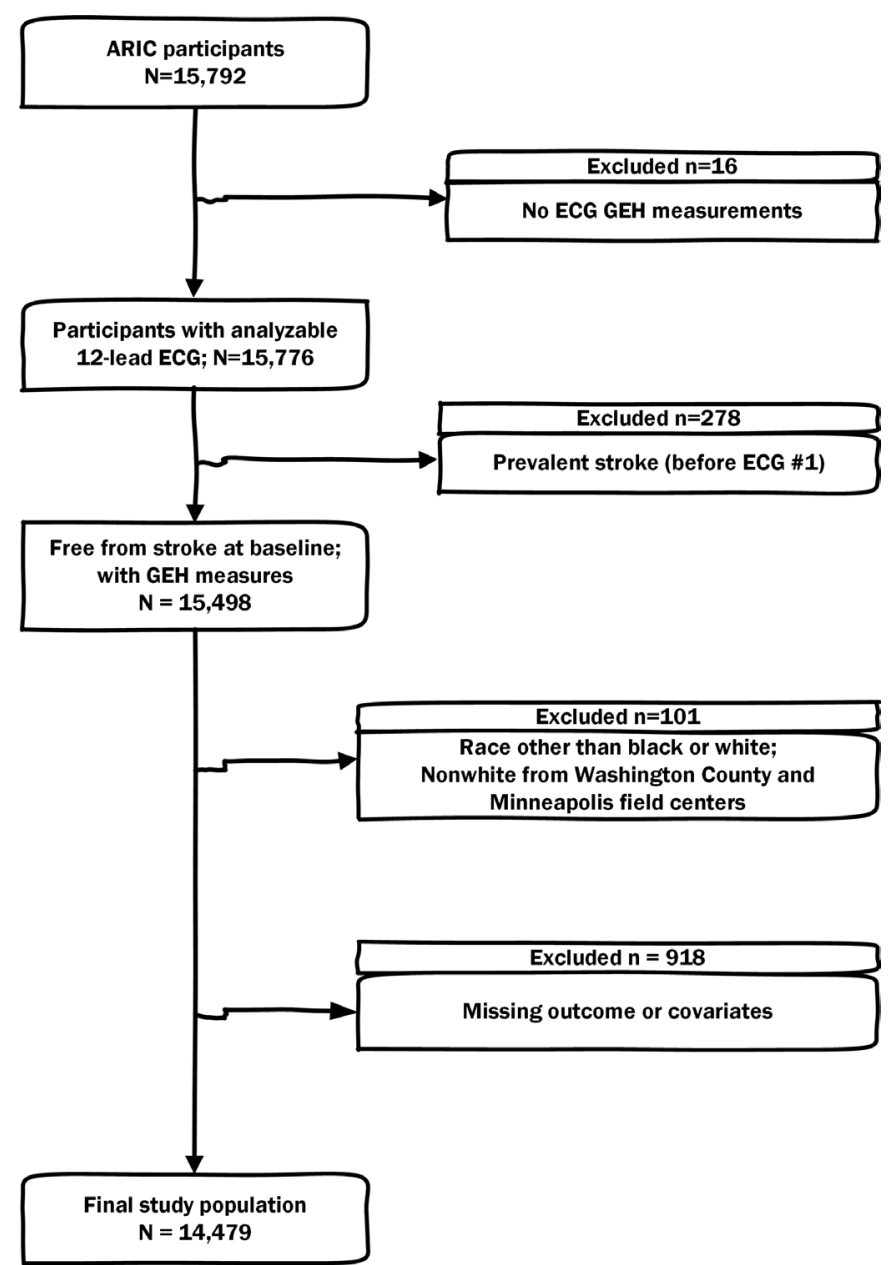

Figure 1 Flow chart of study cohort development. ARIC, Atherosclerosis Risk in Communities; GEH, global electrical heterogeneity.

$(\mathrm{n}=278)$. Further exclusion criteria are shown in figure 1 . The final sample included 14479 participants.

\section{Exposure: GEH and other measures of ventricular substrate}

We analysed resting 12-lead ECGs of the first five study visits. ${ }^{13}{ }^{19-21}$ Visit 1 was coordinated in 1987-1989, visit 2 in 1990-1992, visit 3 in 1993-1995, visit 4 in 1996-1998 and visit 5 in 2011-2013. Electrophysiological ventricular substrate was characterised by novel ECG measures $(\mathrm{GEH})$, by the presence of premature ventricular complexes (PVCs) on 10 s 12-lead ECG, and by traditional ECG measures (QRS duration, Bazett-corrected QTc interval, electrocardiographic LVH (ECG-LVH), bundle branch block (BBB) or interventricular conduction delay (IVCD)).

Each beat on a 10s 12-lead ECG was manually labelled by at least two physicians or physicians-in-training investigators (JAJ, KL, KP, KKP, NEC, LFH, MK-K and LGT) at the Oregon Health \& Science University (Tereshchenko laboratory, $)^{19-21}$ and PVCs were identified. We created a time-coherent median beat with identified isoelectric heart vector origin point. ${ }^{22} \mathrm{~A}$ median beat was comprised of only one (dominant) type. In this study, we included three groups of median beats. Normal (N) group 
included median beats conducting from atria to ventricles (normal sinus, atrial paced, junctional and ectopic atrial median beats). The ventricular (V) group included median beats with ventricular activation originating in ventricles (ventricular paced, and both atrial and ventricular paced median beats). The supraventricular (S) group included median beats of AF or atrial flutter with an atria-to-ventricle type of ventricular conduction.

GEH was measured by spatial QRS-T angle, sum absolute QRST integral (SAIQRST), and SVG magnitude, azimuth, and elevation. ${ }^{23}$ We used two approaches for measurement of SVG vectors and QRS-T angles: areabased and peak-based. ${ }^{1922} 23$ The open-source MATLAB (MathWorks, Natick, Massachusetts, USA) software code for GEH and the heart vector origin measurement is provided at https://physionet.org/physiotools/geh and https://githubcom/Tereshchenkolab/Origin.

QRS duration, QTc, $\mathrm{R}_{\mathrm{aVL}}$ and $\mathrm{S}_{\mathrm{V} 3}$ amplitudes were measured by the $12 \mathrm{SL}$ algorithm as implemented in the Magellan ECG Research Workstation V2 (GE Marquette Electronics, Milwaukee, Wisconsin, USA). Cornell voltage was calculated as the sum of $\mathrm{R}_{\mathrm{avL}}$ and $\mathrm{S}_{\mathrm{v} 3}$ amplitudes. ECG-LVH was defined as a sex-adjusted Cornell product (Cornell voltage times QRS duration) ${ }^{24}>2440 \mathrm{~mm} \times \mathrm{ms}$.

BBB was identified by the Minnesota Code $^{25}$ (left BBB code 7.1, right $\mathrm{BBB}$ code 7.2 and 7.3, fascicular block code 7.6-7.7, bifascicular block code 7.8). IVCD was defined as a QRS duration above $120 \mathrm{~ms}$ in the absence of BBB criteria.

Tachycardia-dependent intermittent BBB (TD-IBBB) was diagnosed on a 12-lead ECG if an intermittent BBB morphology appeared on a normal sinus beat with shortened RR' interval or premature atrial complexe (PAC) with aberrant ventricular conduction (online supplemental figures 1 and 2).

\section{The outcomes: incident stroke subtypes}

The follow-up procedures of ARIC study participants ${ }^{26}$ have been described previously. In this study, we included the follow-up period from 1 January 1987 to 31 December 2018, for participants enrolled in Forsyth County, Minneapolis and Washington County field centres. The follow-up period from 1 January 1987 to 31 December 31st, 2017, was included for participants enrolled in the city of Jackson field centre. Methods for the ascertainment of stroke events have been previously described. ${ }^{27}$ Briefly, records for possible stroke-related hospitalisations were selected based on the International Classification of Diseases, ninth Revision (ICD-9) codes 430-438 until 1997, ICD-9 codes 430-436 and ICD-10 codes G45.X, I60.X, I61.X, I62.X, I63.X, I65.X, I66.X and I67.X afterward. Data on fatal stroke were collected through linkage with the National Death Index. Strokes were then identified by a computer algorithm and adjudicated by physician reviewers. ${ }^{27}$

In the current study, we included three incident stroke subtype outcomes: (1) a first definite or probable thrombotic cerebral infarction (thrombotic stroke, TS), (2) a first definite or probable non-carotid cardioembolic brain infarction (embolic stroke, ES) and (3) an only definite intracerebral haemorrhage (ICH). If a stroke case met the criteria for two ischaemic stroke categories, the following hierarchy was used: haemorrhagic above cardioembolic, above thrombotic, as previously described.$^{27}$ In this study, we considered multiple stroke events for the same participant. Thus, a participant could develop all three incident stroke subtype outcomes as three separate events.

\section{AF, ectopic atrial complexes and use of anticoagulants/aspirin}

Prevalent AF included AF or atrial flutter diagnosed on the first ECG. Incident AF included AF detected on follow-up 12-lead ECG or hospital discharge records and death certificates with ICD-9-CM code 427.31 or 427.32 or ICD-10 code I48 listed in any position. ${ }^{28} 29$

PACs were identified on 10s 12-lead ECG, as described above.

The ARIC study participants were asked to bring their medications to the clinic, where ARIC staff filled out the Medication Survey Form (based partly on self-report by the participants). The use of anticoagulant and aspirincontaining medications in the past 2 weeks was selfreported and validated by medication inventory at every study visit.

\section{Measures of an atrial substrate and use of antiarrhythmic medications}

The atrial substrate was characterised by the following $\mathrm{P}$-wave indices: frontal $\mathrm{P}$ axis, P-terminal force in lead V1 $\left(\mathrm{PTF}_{\mathrm{V} 1}\right)$ and PR interval duration. All P-wave indices, including amplitude and duration of the terminal negative phase (P-prime) of biphasic (positive/negative) $\mathrm{P}$ wave in lead V1, were automatically measured by the 12SL algorithm (GE Marquette Electronics, Milwaukee, WI). $\mathrm{PTF}_{\mathrm{V} 1}$ was calculated as a product of P-prime amplitude and duration. ${ }^{30} 31$

The use of antiarrhythmic medications included in the study was self-reported and subsequently validated by medication inventory. Antiarrhythmic medications included class I, II (beta-blockers), III, IV (phenylalkylamines and benzothiazepines calcium channel blockers) or V (digoxin) antiarrhythmic agents.

\section{Carotid artery plaque}

Carotid artery intima-media thickness was measured in three locations of the carotid arteries bilaterally; at the extracranial carotid artery $1 \mathrm{~cm}$ proximal to the dilatation of the carotid bulb, the carotid bifurcation $1 \mathrm{~cm}$ proximal to the flow divider, and the internal carotid artery $1 \mathrm{~cm}$ distal to the flow divider, using high-resolution B-mode ultrasound (Biosound 2000 II SA; Biosound, Indianapolis, Indiana, USA), as previously described. ${ }^{32-34}$ ARIC ultrasound readers considered an atherosclerotic plaque to be present at any of the six segments if wall thickness exceeded $1.5 \mathrm{~mm}$, or if there was a lumen encroachment or irregular intimal surface or image characteristics indicative of structural heterogeneity of the arterial wall. 
Prevalent cardiovascular disease and common cardiovascular risk factors

The baseline prevalence of cardiovascular disease (CVD) was defined as the presence of prevalent coronary heart disease (CHD), peripheral artery disease (PAD) or heart failure (HF) at the first visit. Prevalent CHD included a self-reported physician-diagnosed heart attack, baseline ECG evidence of myocardial infarction by the Minnesota code, ${ }^{35}$ or a history of coronary revascularisation (either via coronary artery bypass surgery or percutaneous coronary intervention). Prevalent PAD was defined as self-reported history of leg pain during walking that disappeared within $10 \mathrm{~min}$ after rest, an Ankle-Brachial Index $<0.9$ or leg artery revascularisation. ${ }^{36}$ Prevalent HF was defined as asymptomatic (stage 3 by the Gothenburg criteria) HF, manifesting by cardiac and pulmonary symptoms, on medical treatment ${ }^{37}$ or self-reported use of $\mathrm{HF}$ medication.

Body mass index (BMI) was calculated as weight $(\mathrm{kg}) /$ height $(\mathrm{m})^{2}$. Waist-to-hip ratio (WHR) was calculated as a measure of fat distribution. During the clinic visit, sitting blood pressure was measured three times, each after $5 \mathrm{~min}$ of rest. The average of the second and third of three consecutive measurements was used to calculate systolic and diastolic blood pressure levels. Hypertension was defined as blood pressure of $\geq 140 / 90 \mathrm{~mm} \mathrm{Hg}$, or selfreported use of antihypertensive drugs. Self-reported use of antihypertensive and lipid-lowering medications in the past 2 weeks was validated by medication inventory at every study visit. Diabetes was defined as nonfasting blood glucose $\geq 200 \mathrm{mg} / \mathrm{dL}$, fasting blood glucose $\geq 126 \mathrm{mg}$ / $\mathrm{dL}$, self-reported physician diagnosis of diabetes or selfreported use of drugs to treat diabetes. Kidney function was assessed by estimated glomerular filtration rate (eGFR) calculated using the chronic kidney disease (CKD) Epidemiology Collaboration equation (CKDEPI) ${ }^{38}$ Physical activity was measured during leisure time, using the semicontinuous indices ranging from 1 (low) to 5 (high), defined by modified Baecke questionnaire. ${ }^{39}$ Participants self-reported smoking and consumption of alcoholic beverages.

\section{Statistical analyses}

Continuous variables were presented as means and SD. We used analysis of variance (for normally distributed continuous variables) and $\chi^{2}$ test (for categorical variables) to compare baseline clinical characteristics in participants with $0-5$ abnormal GEH parameters.

\section{Analysis of circular variables}

An unadjusted comparison of circular variables (spatial QRS-T angle, SVG azimuth and SVG elevation) was performed using the Mardia-Watson-Wheeler test.

Because distributions of QRS-T and SVG elevation angles were normal or nearly normal, we included them in all conventional statistical analyses without transformation. The SVG azimuth angle was transformed ${ }^{40}$ by doubling its value and then adding $360^{\circ}$.

\section{Survival analyses}

We built cause-specific Cox proportional hazard risk models. Competing other-than-stroke death was censored at the date of death. All continuous ECG exposure variables were expressed as their $\mathrm{z}$ score to standardise comparisons. The proportional-hazards assumption was verified using stcox $\mathrm{PH}$-assumptions suite of tests implemented in STATA (StataCorp), and exceptions were reported. To adjust for confounders, we constructed five models with incremental adjustment. Model 1 was adjusted for demographic characteristics (age, sex and race-study centre group).

Model 2, in addition to model 1 covariates, was adjusted for prevalent at baseline CVD and risk factors (BMI, WHR, total cholesterol, HDL, triglyceride level, use of lipidlowering medications, current smoking, consumption of alcohol, level of physical activity at leisure time, diabetes, hypertension, levels of systolic and diastolic blood pressure, use of blood pressure-lowering drugs, eGFR and the presence of carotid artery plaque).

Model 3 further added characteristics of an atrial substrate (abnormal $\mathrm{P}$ axis (less than 0 or above $75^{\circ}$ ), ${ }^{6}$ abnormal PR interval (less than $120 \mathrm{~ms}$ or above 200 $\mathrm{ms}),{ }^{29}$ abnormal PTF $\left.(\leq-4000 \mu \mathrm{V} \times \mathrm{ms})^{6}\right)$, heart rate, use of antiarrhythmic drugs, anticoagulants and aspirin. In cases of P-wave absence, PR interval and P-wave indices were categorised as abnormal. Model 3 also added presence of PACs on 10s 12-lead ECG at any visit, S or V median beat at any visit and presence of AF at baseline or at any time during follow-up (assuming incident AF has been present since baseline). ${ }^{41}$

Model 4 adjusted for model 3 covariates plus characteristics of an electrophysiological ventricular substrate (QRS duration, QTc interval, presence of BBB/IVCD, ECG-LVH, categorical GEH risk score and presence of PVCs on 10s 12-lead ECG at any visit). Categorical GEH risk score was not included in the models with continuous GEH variables serving as predictors.

Time-updated model 5 for TS and ES outcomes included all model 4 covariates, as well as ECG variables that were updated at the date of ECG recording in visits $1-5$, as time-updated exposure (QRS duration, QTc interval, presence of BBB/IVCD, ECG-LVH and GEH variables), and time-updated covariates (abnormal P-axis, abnormal PTF $_{\mathrm{V} 1}$ abnormal PR interval, heart rate, presence of PACs and PVCs on a single 10s 12-lead ECG and a type of median beat).

Time-updated model 5 for ICH outcome included age, sex, race-study centre group, baseline prevalent CVD and use of antiarrhythmic drugs, anticoagulants and aspirin, time-updated exposure (QRS duration, QTc interval, presence of BBB/IVCD, ECG-LVH and GEH variables one-by-one) and time-updated covariates (abnormal P-axis, PTF ${ }_{\mathrm{V} 1}$ PR interval, heart rate, presence of PACs/ PVCs on a 12-lead ECG and a type of median beat).

Associations of continuous ECG variables with stroke were also studied using adjusted (model 1) Cox regression models incorporating cubic splines with four knots. 
Competing risks of stroke (including fatal stroke) and death from other causes

As the risk of stroke (including fatal stroke) competes with the risk of death from other causes, we employed cause-specific hazards functions, estimated using Cox proportional hazards models. We compared the strength of association of the exposure variables between the two competing outcomes by testing the null hypothesis that the coefficients for a given variable $\left(b_{1}\right.$ and $\left.b_{2}\right)$ were the same across the two competing outcomes by calculating Z-scores:

$$
z=\frac{b_{1}-b_{2}}{\sqrt{\left[\text { s.e. }\left(b_{1}\right)\right]^{2}+\left[\text { s.e. }\left(b_{2}\right)\right]^{2}}}
$$

where s.e is a standard error. We then determined statistical significance of Z-scores.

\section{Subgroups analysis}

We investigated whether sex and age modify the association of electrophysiological ventricular substrate with incident stroke by adding interaction terms in the fully adjusted Cox model 5. Separate models were constructed for each outcome, including the interaction terms of ECG variables with (1) sex and (2) age as a continuous variable.

\section{Sensitivity analyses}

To assess the robustness of the study findings, we conducted sensitivity analysis after excluding participants with different types of stroke.

To determine the additive predictive value in comparison with the $\mathrm{CHA}_{2} \mathrm{DS}_{2}$-VASc risk score, we studied an incident AF cohort in our study. From our main study population $(n=14479)$, we excluded participants with prevalent AF $(n=26)$, without incident AF during follow-up $(n=11178)$, and missing incident AF status in $2017(n=264)$. The remaining 3011 participants with incident AF were included. The $\mathrm{CHA}_{2} \mathrm{DS}_{2}$-VASc score was calculated at the date of incident AF diagnosis. The exposure ECG variables were taken as measured prior (as close as possible) to incident AF. Time at risk was calculated from the date of AF ascertainment until the date of ischaemic stroke, other-than-stroke death or lost to follow-up, whichever occurred first. To compare the predictive value of GEH for ischaemic stroke, we compared the performance of $\mathrm{CHA}_{2} \mathrm{DS}_{2}$-VASc score alone and after addition of $\mathrm{GEH}$ variables. We assessed model performance by C-statistic, relative integrated discrimination improvement (IDI) and categorical net reclassification improvement (NRI) for categories of $<1 \%, 1 \%-2 \%$ and $>2 \%$ 1-year risk of stroke.

Statistical analyses were performed using STATA MP V.16.1 (StataCorp). A p $<0.05$ was considered statistically significant. We used the Strengthening the Reporting of Observational Studies in Epidemiology (STROBE) crosssectional checklist when writing our report. ${ }^{42}$

\section{Patient and public involvement}

Patients or the public were not involved in the design, conduct or reporting of this study.

\section{RESULTS}

\section{Study population}

More than half of the participants had 0-1 abnormal GEH measures, and only 6\% had 4-5 abnormal GEH metrics (online supplemental table 1). PVCs were detected in $280(1.93 \%)$ participants at baseline and 1202 $(8.30 \%)$ participants at any visit. TD-IBBB was observed in at least one ECG in $122(0.84 \%)$ participants. There was a gradual increase in the presence of carotid plaque and a gradual decrease in the level of HDL, consistent with a growth in the number of abnormal GEH metrics. As expected, abnormal $\mathrm{P}$ wave indices, PACs, PVCs and ventricular conduction abnormalities were more likely to be observed in participants with abnormal GEH.

\section{Incident stroke}

Over a median follow-up of 24.5 years, there were 899 TS (incidence 2.87; 95\% CI 2.69 to 3.07 per 1000 personyears), 400 ES (incidence 1.26; 95\% CI 1.14 to 1.39 per 1000 person-years) and 120 ICH-only strokes (incidence $0.38 ; 95 \%$ CI 0.32 to 0.45 per 1000 person-years). Both types of ischaemic stroke were diagnosed in 62 participants, and another 16 participants endured both ICH and either TS or ES. Baseline GEH was worse in participants who developed any type of stroke during follow-up (table 1).

\section{Electrophysiological ventricular substrate and cardio-ES}

In minimally adjusted model 1 , nearly all measures of the electrophysiological ventricular substrate were associated with incident ES (table 2). Model 2 showed that CVD and cardiovascular risk factors explained a significant portion of it (figure 2), but an association of SAIQRST and PVCs with ES remained significant.

The addition of the atrial substrate metrics in model 3 resulted in further attenuation of the association of QRS-T angle and PVCs with ES. Model 4 confirmed that spatial QRS-T angle, SAIQRST and PVCs represent specific features of the electrophysiological ventricular substrate independently associated with ES. Notably, the association of QRS-T angle and SAIQRST with ES was 'dose-dependent,' as demonstrated by the gradually increasing risk of ES across the distribution of QRS-T angle and SAIQRST (figure 3). In time-updated model 5, SVG magnitude emerged as significant independent transient exposure associated with incident ES.

Sensitivity analysis in participants with pure ES (online supplemental table 2) reported consistent results.

\section{Electrophysiological ventricular substrate and TS}

In minimally adjusted model 1 , nearly all traditional and novel measures of the ventricular substrate associated with TS, although the strength of association was weaker 


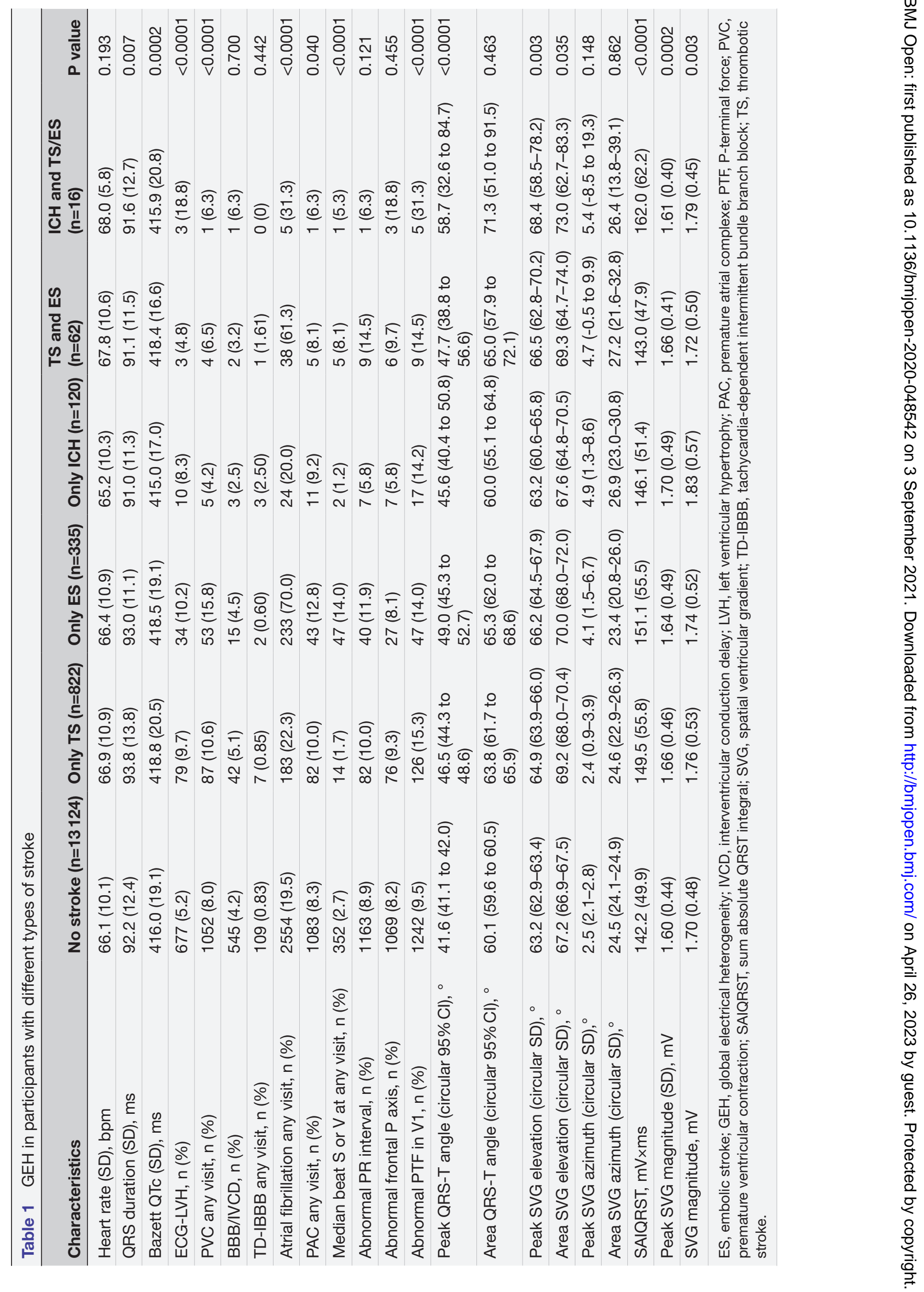




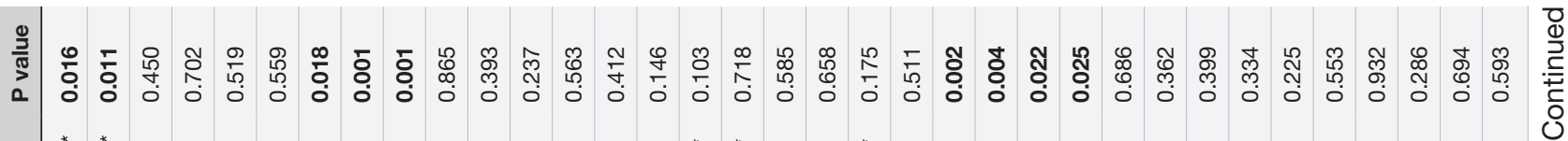

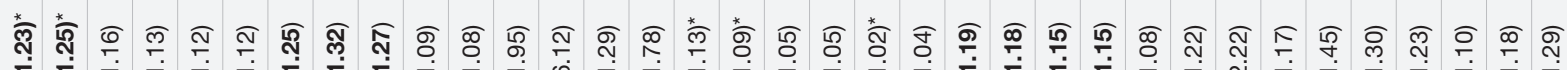

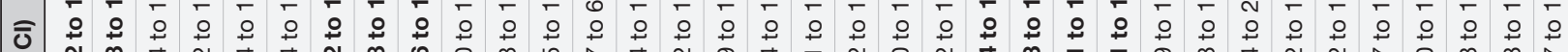
L 可

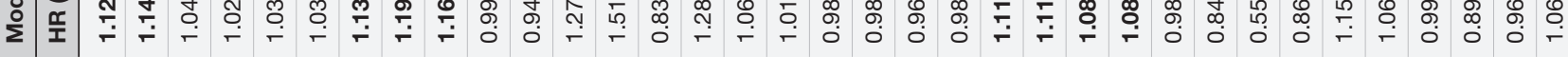

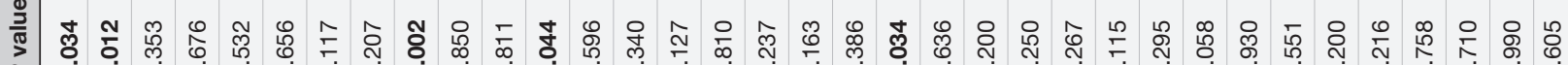
a

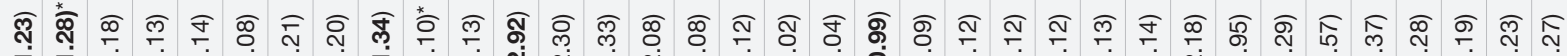

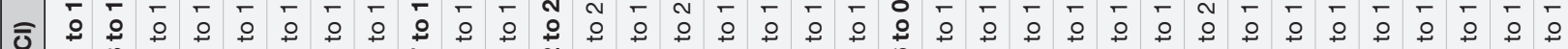

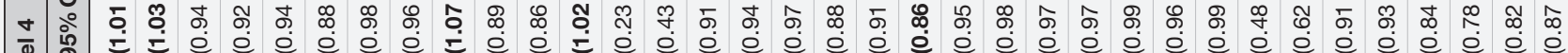
d

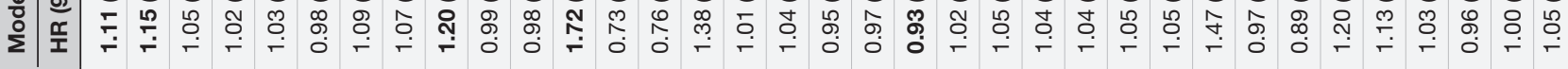

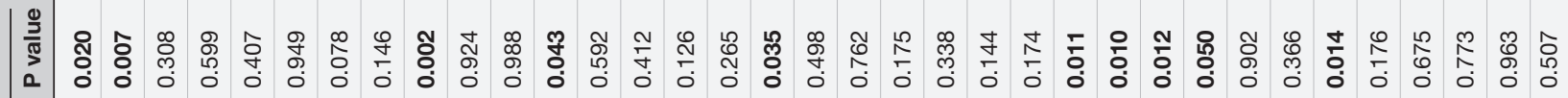

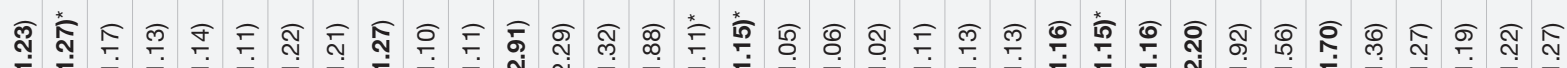

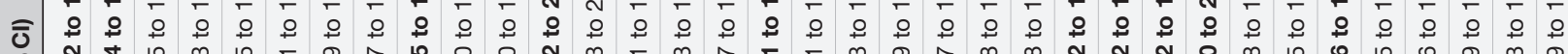

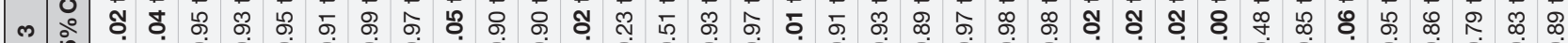
ब岁 送

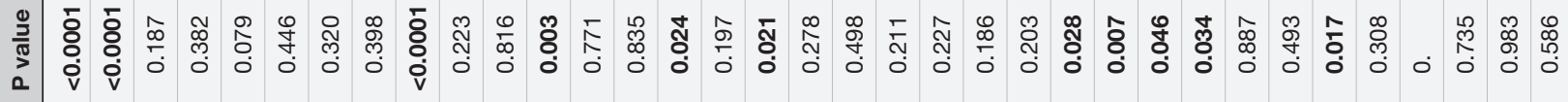

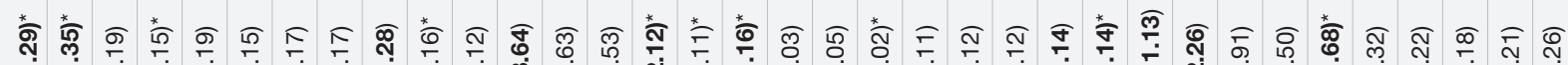

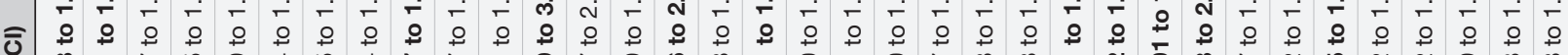
N

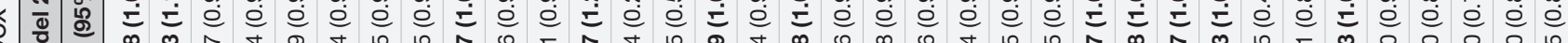

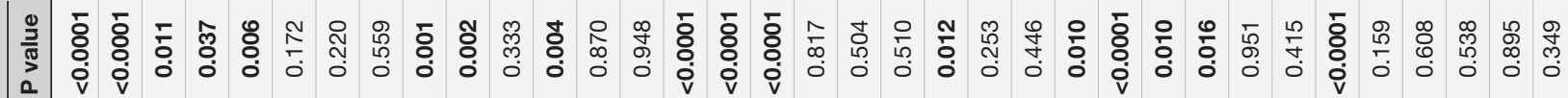

苟 品

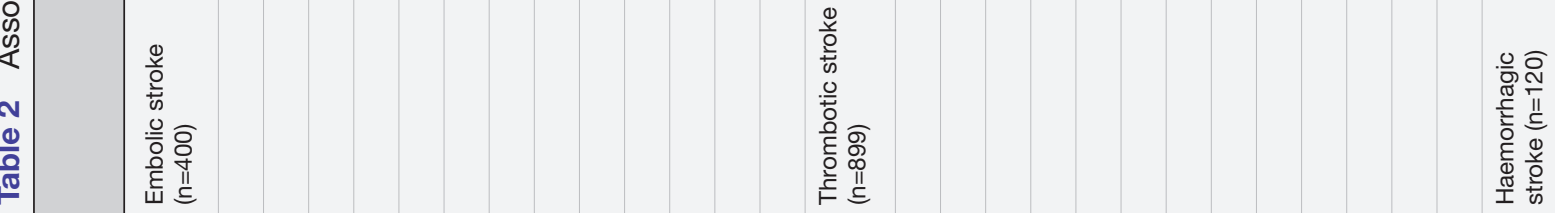




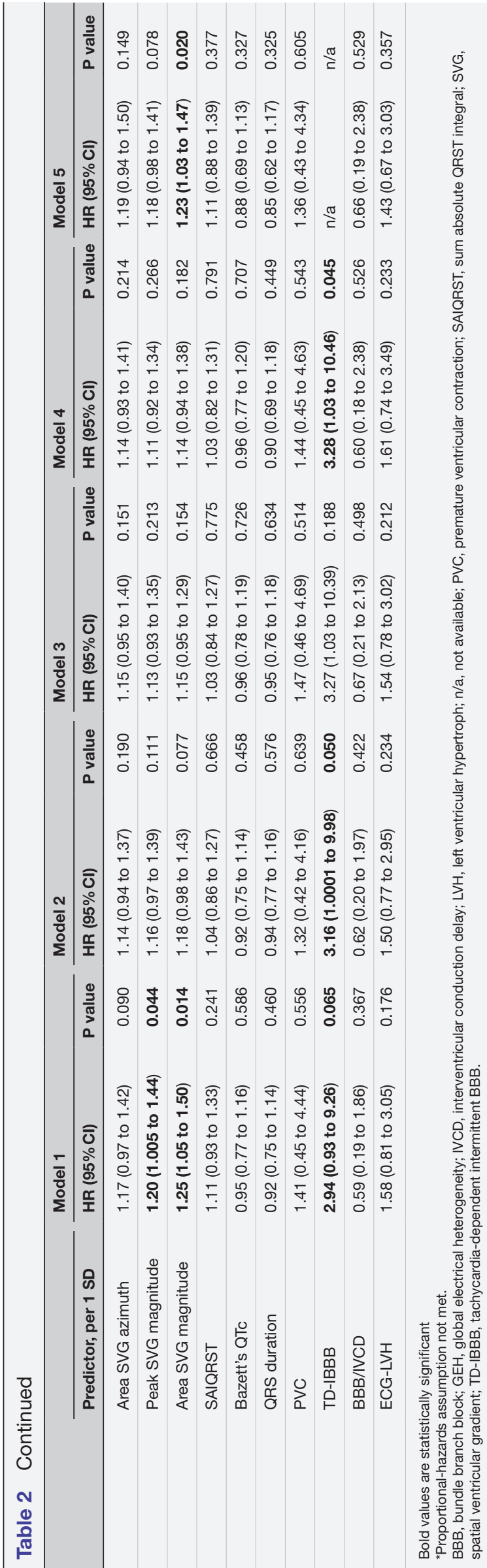

for thrombotic than cardio-ES (table 2). Similar to ES, the risk of TS increased across the distribution of the QRS-T angle and SAIQRST (figure 3). Adjustment for CVD and cardiovascular risk factors in model 2 substantially attenuated the association for all ECG metrics (figure 2).

However, in contrast to ES, further adjustment for atrial substrate in model 3 did not change the strength of associations, suggesting that an association of a ventricular substrate with TS was independent of atrial substrate. Again, in contrast to the ES, model 4 showed that there was no independent association of the electrophysiological ventricular substrate with incident TS, as previously observed in models 1-3 associations attenuated and were not statistically significant.

Interestingly, time-updated model 5 revealed a strengthened association of SAIQRST and SVG magnitude with TS, suggesting the importance of time-dependent transient electrophysiological ventricular substrate (figures 2 and 4).

\section{Electrophysiological ventricular substrate and haemorrhagic stroke}

In contrast to ischaemic stroke, only time-updated SVG magnitude (figure 4) and detected at any visit TD-IBBB (model 4, HR 3.28, 95\% CI 1.03 to 10.46 ; $\mathrm{p}=0.045$ ). were associated with ICH.

Sensitivity analysis with pure TS (online supplemental table 2) demonstrated identical results, supporting the robustness of the study findings.

\section{Competing other-then-stroke death}

There were 4417 deaths from other-than-stroke causes (incidence 14.78 ; $95 \%$ CI 14.35 to 15.22 ) per 1000 personyears of follow-up. Most ECG variables had similar associations with both competing outcomes, with few notable exceptions.

Peak SVG magnitude demonstrated statistically significant discordant association with competing outcomes: larger SVG magnitude was associated with a higher risk of all types of incident stroke but lower risk of stroke-free death (online supplemental table 3). Similarly, TD-IBBB was associated with a greater risk of ICH but not strokefree death. Consistently, PVC's presence was stronger associated with ischaemic stroke than with competing stroke-free death.

\section{Subgroups analysis}

We observed a few statistically significant interactions (online supplemental table 4). The presence of PVCs was associated with a twofold higher risk of ES in women compared with men (relative HR (RHR) 2.2; 95\% CI 1.0 to 5.0; $\mathrm{p}=0.051$ ). Peak SVG elevation displayed discordant association with TS in men and women. More superiorly directed SVG vector was associated with a lesser risk of TS in women but higher risk of TS in men.

ECG-LVH was associated with a fivefold higher ICH risk in men than women (RHR 5.6; 95\% CI 1.1 to 28.5; $\mathrm{p}=0.039$ ). The association of age with incident ICH was 
Embolic Stroke

Thrombotic Stroke

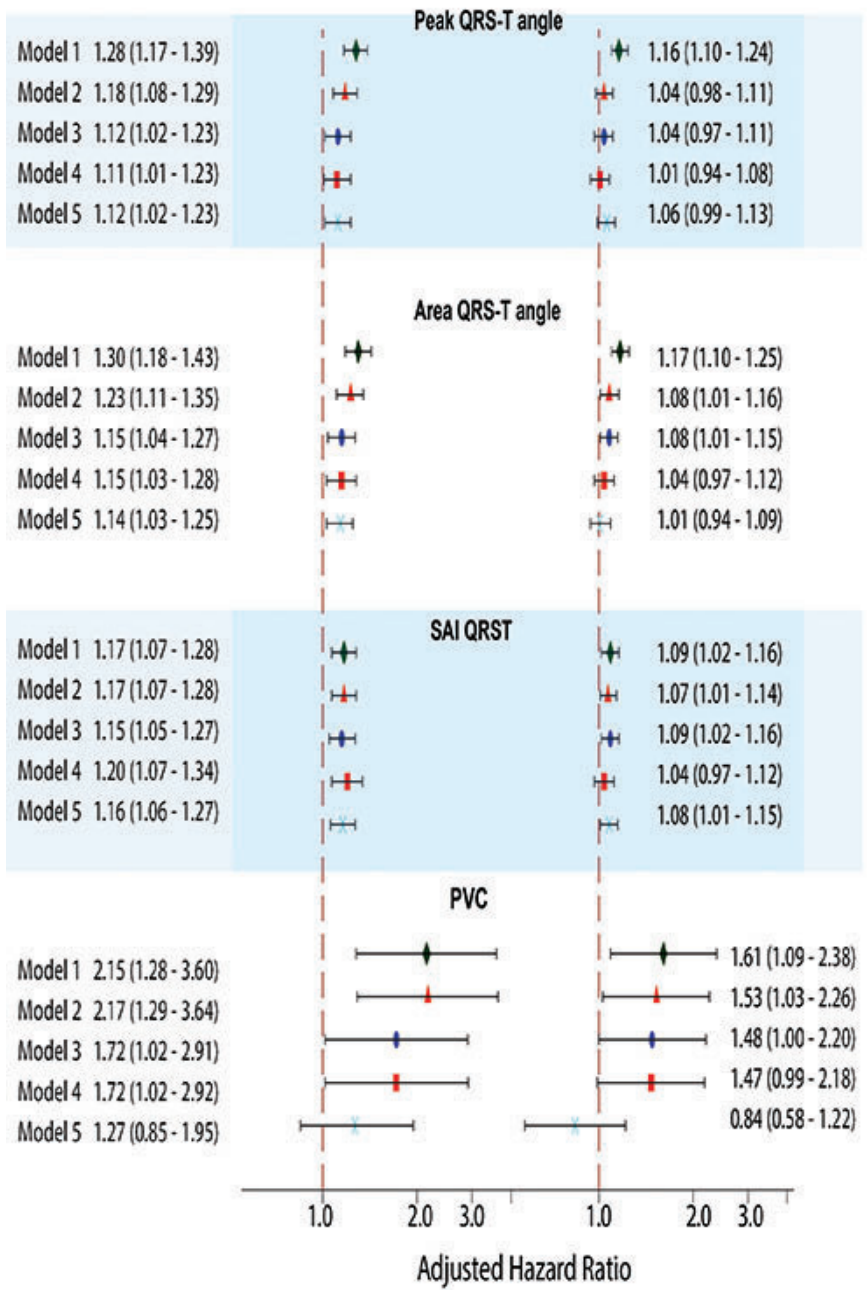

Figure 2 Adjusted Cox proportional HR with 95\% Cl for the association of peak and area QRS-T angle, SAIQRST and a PVC on a 10 s ECG with ES and TS. Black lines correspond to $95 \% \mathrm{Cl}$ bounds. Model 1 (green diamond) was adjusted for age, sex and race study centre. Model 2 (orange triangle) was in addition adjusted for prevalent CVD and risk factors (BMI, WHR, lipids, use of lipid-lowering medications, smoking, alcohol use, physical activity, diabetes, hypertension, systolic and diastolic blood pressure, use of antihypertensive drugs, eGFR ${ }_{\text {CKD-EPI, }}$ carotid artery plaque). Model 3 (blue oval) added adjustment for atrial substrate (abnormal P axis, PR interval, PTF $_{\mathrm{V} 1}$ ), heart rate, antiarrhythmic drugs, anticoagulants, aspirin, PACs on 12-lead ECG at any visit, S or V median beat at any visit and AF. Model 4 (red rectangle) further added electrophysiological ventricular substrate (QRS duration, QTC interval, BBB/IVCD, ECG-LVH and PVCs on 12-lead ECG at any visit). Time-updated model 5 (cyan X) added ECG variables that were updated at the date of ECG recording in visits 1-5, as time-updated exposure (QRS duration, QTc interval, presence of BBB/IVCD, ECG-LVH and GEH) and time-updated covariates (abnormal P-axis, PTF ${ }_{\mathrm{v} 1}$, PR interval, heart rate, PACs or PVCs on a 12-lead ECG and a type of median beat). AF, atrial fibrillation; BBB, bundle branch block; BMI, body mass index; eGFR, estimated glomerular filtration rate; ES, embolic stroke; GEH, global electrical heterogeneity; IVCD, interventricular conduction delay; LVH, left ventricular hypertrophy; PACs, premature atrial complexes; PTF ${ }_{\mathrm{V} 1}$, P-terminal force in lead V1; PVC, premature ventricular contraction; SAIQRST, sum absolute QRST integral; TS, thrombotic stroke; WHR, waist-to-hip ratio.

modified by QRS-T angle (online supplemental figure 3) and SVG azimuth (online supplemental figure 4). The risk of $\mathrm{ICH}$ was rising steeper with age if the baseline QRS-T angle was smaller, and SVG azimuth was directed more anteriorly.

\section{Reclassification improvement in incident AF patients}

Out of 3011 ARIC participants with incident AF, there were 204 ischaemic strokes (either ES or TS) within the first year after AF diagnosis. The addition of all GEH variables to $\mathrm{CHA}_{2} \mathrm{DS}_{2}$-VASc score improved the C-statistic $(95 \% \mathrm{CI})$ from 0.560 (0.515 to 0.604$)$ to 0.605 (0.561 to $0.650) ; \mathrm{p}=0.005$. Reclassification was also improved: relative IDI was 1.31 (95\% CI 0.81 to 1.79$)$; $\mathrm{p}<0.0001$. Categorical NRI was 0.282 (95\% CI 0.182 to 0.381$) ; \mathrm{p}<0.0001$. Out of participants with ischaemic stroke, $62 \%$ were appropriately reclassified to higher risk categories. Out of stroke-free participants within 1 year, $53 \%$ were appropriately reclassified to lower-risk categories. 

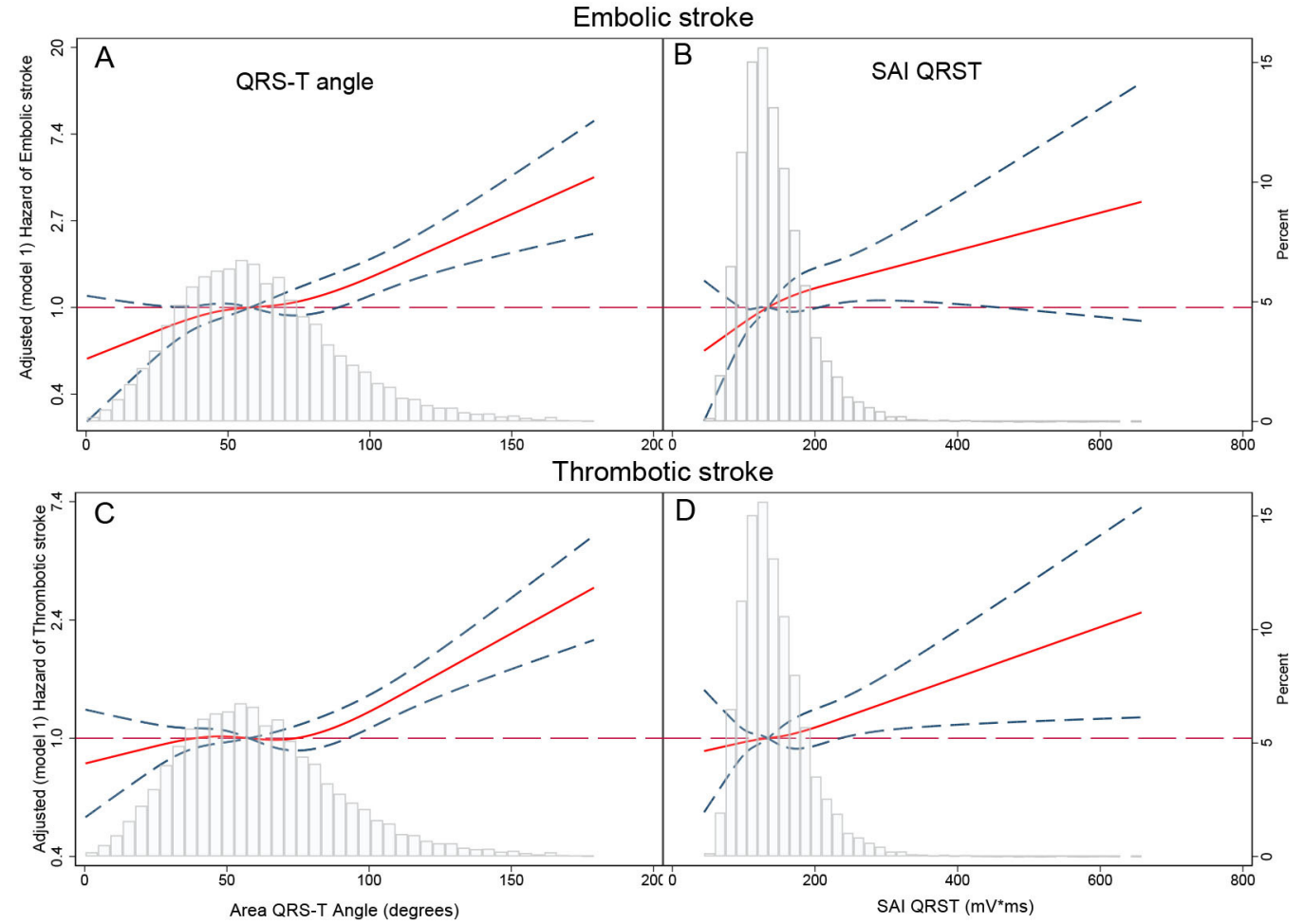

Figure 3 Adjusted (model 1) risk of cardioembolic (A, B) and thrombotic (C, D) stroke associated with area QRS-T angle (A, C) and SAIQRST (B, D). Restricted cubic spline with $95 \% \mathrm{Cl}$ shows a change in the HR (y-axis) in response to QRS-T angle and SAIQRST change (x-axis). 50th percentile of QRS-T angle and SAIQRST is selected as a reference. Knots of area QRS-T angle are at $21^{\circ}-48^{\circ}$ to $69^{\circ}-114^{\circ}$. Knots of SAIQRST are at $83-119$ to $151-230 \mathrm{mV} \times \mathrm{ms}$. SAIQRST, sum absolute QRST integral.

\section{DISCUSSION}

This large, prospective, community-dwelling cohort study revealed several novel findings. First, we uncovered a novel electrophysiological ventricular substrate of ES, likely reflecting the development of cardiac memory in response to PVCs. Our results suggest that the search for the sources of ES should include an assessment of the PVC burden and open an avenue for randomised clinical trials of the ablation of idiopathic PVCs as a possible prevention strategy for a cardio-ES of uncertain source.

Second, we observed a time-dependent association of a transient electrophysiological ventricular substrate likely reflecting cardiac memory in patients with TS, suggesting that PVCs can also trigger a thromboembolic stroke from a brain artery. Future studies are needed to determine whether monitoring of cardiac memory and PVC burden in patients at risk for TS can be used to guide short-term dual antiplatelet therapy.

Third, we observed important differences between men and women, warranting further investigations. The presence of PVCs was associated with a twofold higher risk of ES in women than men, whereas ECG-LVH was associated with a fivefold higher ICH risk in men than women.

Finally, we found an association of electrophysiological ventricular substrate with ICH, possibly reflecting the development of cardiac memory in response to TD-IBBB and suggesting an implication of amyloidosis. After validation of these findings in another study, TD-IBBB may be used as a marker of ICH risk, increasing clinical suspicion of cardiac amyloidosis.

Notably, our study suggested that the wide spatial QRS-T angle, and the large SAIQRST and SVG magnitude are nonspecific characteristics of the transient substrate of cardiac memory, which develops in response to PVCs and TD-IBBB. Further ECG monitoring studies are needed to validate an association of PVCs and TD-IBBB burden with the amount of cardiac memory.

\section{The electrophysiological ventricular substrate of ischaemic stroke}

$\mathrm{AF}$ is a well-recognised major risk factor and the central focus for cardio-ES prevention. ${ }^{43}$ The pathophysiological mechanisms of stroke in AF have been studied for decades. ${ }^{44}$ However, a frequently missing temporal link between $\mathrm{AF}$ episodes and stroke events remains incompletely understood. ${ }^{45} 46$ The atrial myopathy hypothesis ${ }^{47}$ was developed to explain the temporal dissociation between $\mathrm{AF}$ and stroke. The hypothesis suggests that the fibrotic atrial substrate, which interacts with the atrial electrical dysfunction, ${ }^{48}$ is the principal mediator of thromboembolism. Nevertheless, the risk of stroke is increased in a graded manner in patients with paroxysmal, persistent and permanent AF. ${ }^{49}{ }^{50}$ Moreover, a greater $\mathrm{AF}$ burden is associated with a higher risk of stroke ${ }^{5152}$ highlighting the importance of a haemodynamic disturbance triggering thromboembolic events. Therefore, the current strategy for determining the aetiology of cardio-ES is to search for underlying $\mathrm{AF}^{53}$ 


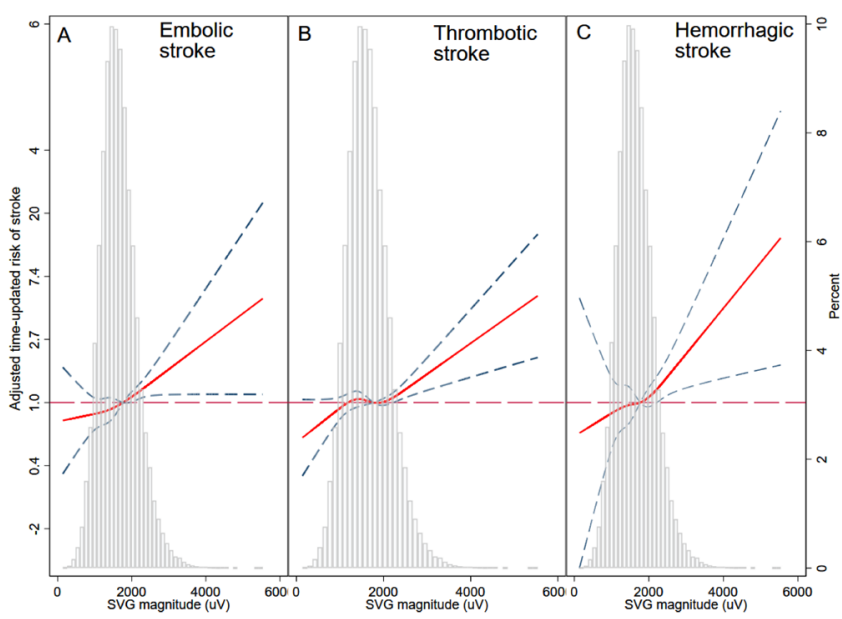

D

Time-updated risk of stroke

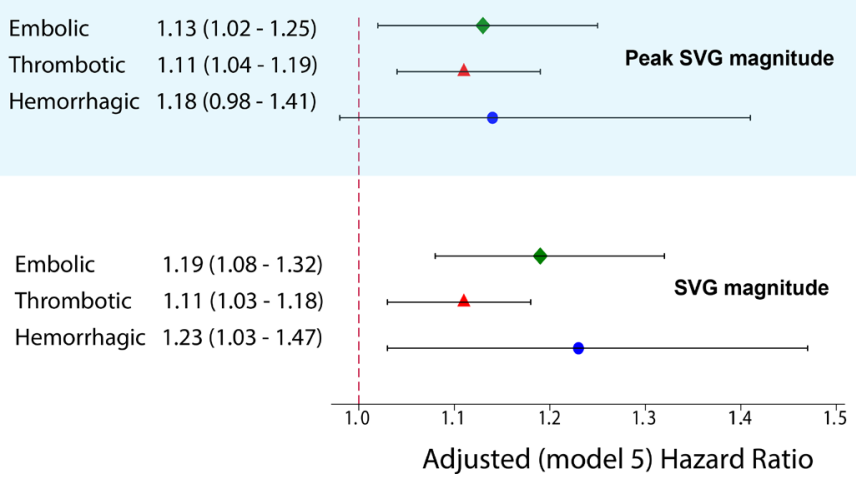

Figure 4 Time-updated adjusted risk of (A) ES, (B) TS and (C) ICH associated with SVG magnitude. Time-updated Cox regression models were adjusted for age, sex, race and study centre, time-updated heart rate, and the type of median beat. Restricted cubic spline with $95 \% \mathrm{Cl}$ shows a change in the HR (y-axis) in response to SVG magnitude change ( $x$ axis). The 50th percentile of SVG magnitude is selected as a reference. Knots of SVG magnitude are at 0.92-1.43 to 1.79$2.51 \mathrm{mV}$. (D). Adjusted Cox proportional HR with $95 \% \mathrm{Cl}$ for the association of SVG magnitude with ES (green diamond), TS (red triangle) and $\mathrm{ICH}$ (blue circle) in time-updated model 5. ES, embolic stroke; ICH, intracerebral haemorrhage; SVG, spatial ventricular gradient; TS, thrombotic stroke.

Our study uncovered the presence of an electrophysiological ventricular substrate associated with ES (figure 5), which remained associated with ES even after rigorous adjustment for CVD and cardiovascular risk factors, AF and atrial substrate, ${ }^{54}$ and known ventricular abnormalities (BBB, LVH). PVC burden, manifested by the cardiac memory, characterises the electrophysiological ventricular substrate of ES. Several previous studies reported an association of PVCs with ischaemic stroke..$^{9-11}$ Previous studies $^{9-11}$ speculated that PVCs merely reflect the presence and burden of CVD. Nevertheless, our study demonstrated that the association of PVCs with ES is independent of CVD and cardiovascular risk factors (model 2, figure 2), as well as traditional CVD-related electrophysiological ventricular substrate (ECG-LVH and BBB; model 4, figure 2).

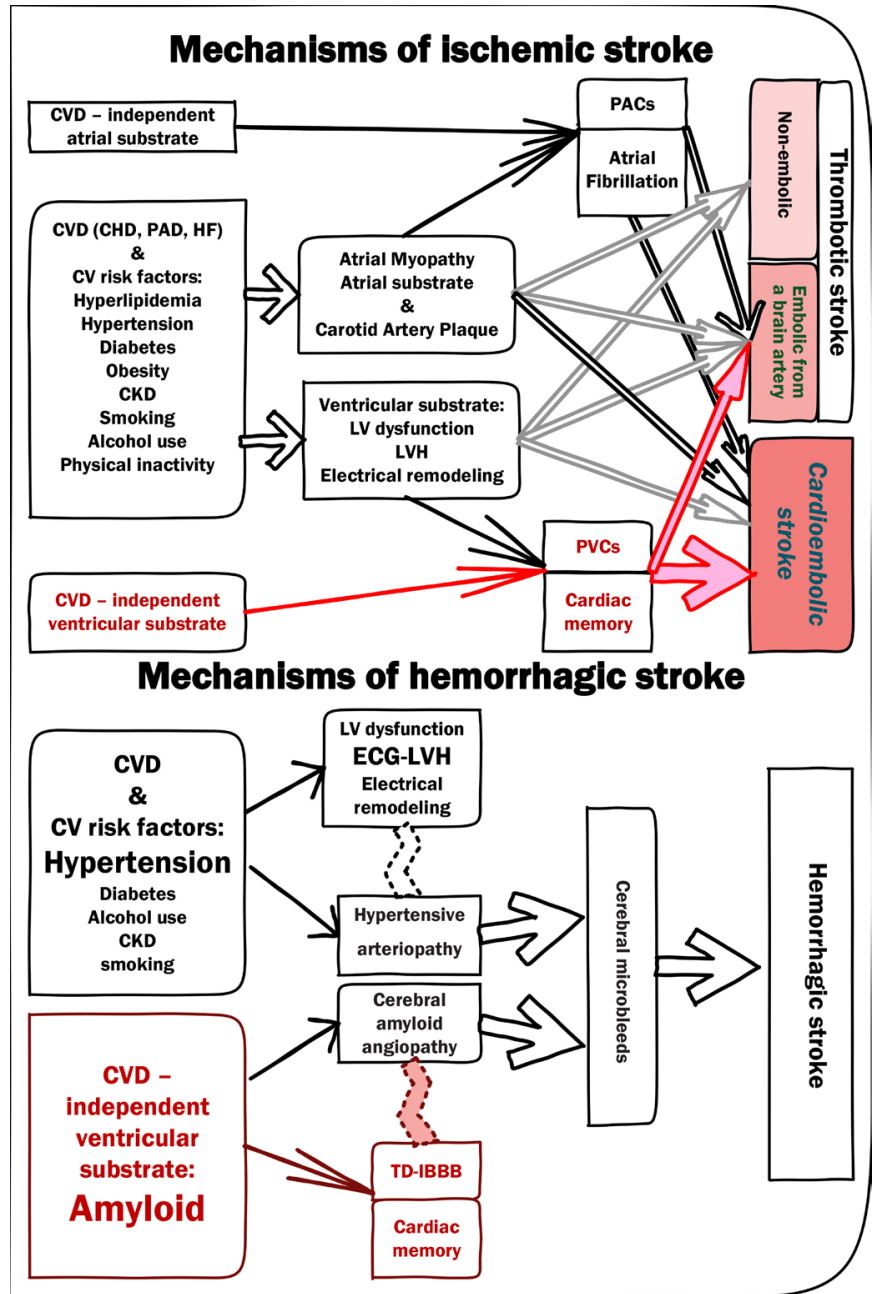

Figure 5 Mechanisms of ischaemic (A) and haemorrhagic (B) stroke. Red colour highlights the mechanisms suggested by this study. CHD, coronary heart disease; CVD, cardiovascular disease; HF, heart failure; LVH, left ventricular hypertrophy; PACs, premature atrial complexes; PAD, peripheral artery disease; PVCs, premature ventricular contractions; TD-IBBB, tachycardia-dependent intermittent bundle branch block.

Moreover, we showed that AF and atrial substrate mediate the association of PVCs with ES (model 3, figure 2).

It is well known that PVCs can induce cardiac memory, which is manifested by a wide QRS-T angle ${ }^{5657}$ and a large QRST integral. ${ }^{58}$ In this study, the response of the specific electrophysiological ventricular substrate measures (PVCs, spatial QRS-T angle and SAIQRST) on stepwise adjustment (models 1-4, figure 2) was similar, supporting the notion that spatial QRS-T angle and SAIQRST reflect cardiac memory. We also observed a gradual, 'dose-dependent' association of QRS-T angle and SAIQRST with the hazard of cardio-ES (figure 3A).

Evidently, several mechanisms behind abnormal QRS-T angle $^{59}$ and SAIQRST ${ }^{60}$ explain an attenuation of the association after adjustment for CVD and cardiovascular risk factors. Importantly, spatial QRS-T angle and SAIQRST remained associated with ES after full adjustment in timeupdated model 5. This raises the possibility that cardiac 
memory reflects an increasing burden of PVCs before the stroke event. Moreover, we observed a strong, independent, 'dose-dependent' association of time-updated SVG magnitude with ES. In competing risk analysis, SVG magnitude was stronger associated with ES than competing death from other causes. SVG magnitude reflects cardiac memory ${ }^{16}$ as a transient substrate or a trigger. ${ }^{19}$ As expected, the SVG direction did not associate with incident stroke because idiopathic PVCs can originate from different locations. ${ }^{59}$ Therefore, the results of our study suggest that the high burden of PVCs can trigger cardioembolic events. Future studies are needed to prove an association of PVC burden with ES.

PVC burden can be a 'missing' temporal link connecting haemodynamic disturbance due to paroxysmal arrhythmia with acute stroke events in predisposed patients (figure 5A). The ablation of idiopathic PVCs could be an effective and safe ${ }^{60}$ prophylactic measure to reduce the risk of acute stroke. Our findings open an avenue for randomised clinical trials of ES prevention using the ablation of idiopathic PVCs, especially for the secondary prevention of an ES of uncertain source.

Consistent with the notion that a subset of TSs includes ES originating from a brain artery, we observed a weak association of PVCs, spatial QRS-T angle, SAIQRST and timeupdated SVG magnitude with TS. As expected and contrary to ES, the association of a ventricular substrate with TS was independent of AF and atrial substrate. Still, the association was mediated primarily by CVD and cardiovascular risk factors, including BBB and LVH.

Similar to ES, a dose-dependent association of spatial QRS-T angle, SAIQRST and time-updated SVG magnitude with the risk of TS suggested a similar underlying mechanism—cardiac memory, reflecting PVC burden. Notably, in competing risk analysis, PVCs and SVG magnitude were stronger associated with TS than competing non-stroke death. However, unlike in ES, an ES from a brain artery seems to be more likely triggered by CVD-related PVCs. Thus, monitoring of SVG magnitude (ie, monitoring of cardiac memory due to PVC burden) in patients at risk of TS can potentially be used to guide short-term dual antiplatelet therapy or timing of carotid endarterectomy, which should be investigated further (figure 5A).

\section{The electrophysiological ventricular substrate in haemorrhagic stroke}

In this study, we observed that transient increases in SVG magnitude and TD-IBBB was associated with ICH. Cardiac memory developed in response to TD-IBBB has been previously demonstrated. ${ }^{61}$ TD-IBBB raises suspicion of amyloidosis (figure $5 \mathrm{~B}$ ).

Cerebral microbleeds are the strongest risk factors of haemorrhagic stroke. ${ }^{62}$ Cerebral amyloid angiopathy is the second most common cause of haemorrhagic stroke, following hypertension (hypertensive arteriopathy) ${ }^{63}$ A previous study demonstrated an association of ECG-LVH with ICH. ${ }^{34}$ We adjusted our analyses by ECG-LVH, history of hypertension, antihypertensive treatment and levels of systolic and diastolic blood pressure to account for hypertensive arteriopathy as the leading cause of ICH.

The amyloid burden is independently associated with cerebral microbleeds. ${ }^{64}$ Amyloidosis is a systemic disease. ${ }^{65}$ Cardiac amyloidosis is significantly underdiagnosed, partly because of a lack of suspicion and a widespread belief that it is rare ${ }^{66}$ Nevertheless, wild-type transthyretin amyloidosis (ATTRwt) cardiac amyloidosis is common in older adults ${ }^{67}$ and patients with HF with preserved ejection fraction. ${ }^{68}$ Recently, novel diagnostic approaches ${ }^{69}$ and therapies ${ }^{70}$ emerged, which can improve clinical outcomes, facilitating a search for early diagnostic clues.

Previously described ECG manifestations of cardiac amyloidosis are highly variable ${ }^{71}$ and include ECG-LVH, low voltage and poor $\mathrm{R}$ wave progression. ${ }^{68}$ Notably, one consistent characteristic feature of all types of cardiac amyloid (light chain, mutant ATTR and ATTRwt) includes cardiac conduction abnormalities. ${ }^{72}$ In this study, individuals with TD-IBBB had a twofold higher risk of ICH, but the association was not statistically significant and should be validated in another independent study. The frequency and timing of TD-IBBB in cardiac amyloidosis should be studied further.

Up to $25 \%$ of patients with cerebral amyloid angiopathy are also diagnosed with $\mathrm{AF}^{73}$ which poses a difficult dilemma and discussion about the risks and benefits of anticoagulation for stroke prevention. ${ }^{74}$ In our study, 16 patients experienced both haemorrhagic and ischaemic stroke. Our results hold a promise that in the future, propensities towards ischaemic and haemorrhagic stroke can be dissected based on the vectorcardiogram (VCG) features of the ventricular substrate.

\section{Conclusions and clinical implications}

While many cardiovascular risk factors of stroke are known, not all stroke mechanisms are recognised. A frequently missing temporal link between AF episodes and cardio-ES events remains incompletely understood. Previous studies reported an association of PVCs and LVH with incident stroke, but the role of cardiac electrophysiological ventricular substrate in different types of stroke was underappreciated.

The current large prospective cohort study uncovered the electrophysiological ventricular substrate of stroke, reflecting the development of cardiac memory. In patients with ischaemic stroke, cardiac memory was developed in response to PVCs. In patients with ICH, cardiac memory was developed in response to the transient TD-IBBB, suggesting an implication of amyloidosis.

Our results suggest that the search for the sources of cardio-ES should include an assessment of the PVC burden. Randomised clinical trials of the ablation of PVCs as a possible prevention strategy for a cardio-ES of the uncertain source should be considered. The monitoring of cardiac memory on ECG may be useful to guide short-term dual antiplatelet therapy for prevention of ischaemic stroke. TD-IBBB should be further studied as 
a marker of ICH risk, increasing clinical suspicion for cardiac amyloidosis.

\section{Twitter Larisa G Tereshchenko @Tereshchenkolab}

Acknowledgements The authors thank the staff and participants of the ARIC study for their important contributions.

Contributors JAJ participated in the study design and drafting of the manuscript. KTH participated in automated ECG analysis software development and conducted automated ECG analysis. KL, KKP, KP, NEC, NWLS, LFH and MK-K analysed ECGs and labeled beats and performed quality control of ECG analyses. All authors interpreted the study findings and revised the manuscript. LGT designed the study, handled the study funding, directed the study implementation, including quality assurance and control, conducted statistical analyses and critically revised the manuscript. All authors approved the submitted version and have agreed both to be personally accountable for the author's own contributions and the accuracy and integrity of any part of the work.

Funding The Atherosclerosis Risk in Communities study has been funded in whole or in part with Federal funds from the National Heart, Lung and Blood Institute, National Institutes of Health, Department of Health and Human Services, under Contract nos. (HHSN268201700001I, HHSN268201700002I, HHSN268201700003I, HHSN268201700004I, HHSN268201700005I). This work was supported by HL118277 (LGT).

Competing interests None declared.

Patient consent for publication Not required.

Ethics approval Informed consent was obtained from all study participants prior to enrollment. This study was approved by the Oregon Health \& Science University Institutional Review Board (IRB00010346). In addition, the ARIC Publication Committee approved this manuscript (ARIC Manuscript \#3333). All procedures performed in studies involving human participants were in accordance with the ethical standards of the Institutional Review Board and the 1964 Declaration of Helsinki and its later amendments or comparable ethical standards.

Provenance and peer review Not commissioned; externally peer reviewed.

Data availability statement Data are available in a public, open access repository. The ARIC Study data are available through the National Heart, Lung, and Blood Institute's Biological Specimen and Data Repository Information Coordinating Center, the National Center of Biotechnology Information's database of Genotypes and Phenotypes, and via the ARIC Coordinating Center at the University of North Carolina-Chapel Hill. The open-source MATLAB (MathWorks, Natick, MA, USA) code for ECG analysis is provided at https://physionet.org/physiotools/geh and https://github.com/Tereshchenkolab/Origin. Statistical analysis code is provided at https://github.com/Tereshchenkolab/statistics.

Supplemental material This content has been supplied by the author(s). It has not been vetted by BMJ Publishing Group Limited (BMJ) and may not have been peer-reviewed. Any opinions or recommendations discussed are solely those of the author(s) and are not endorsed by BMJ. BMJ disclaims all liability and responsibility arising from any reliance placed on the content. Where the content includes any translated material, BMJ does not warrant the accuracy and reliability of the translations (including but not limited to local regulations, clinical guidelines, terminology, drug names and drug dosages), and is not responsible for any error and/or omissions arising from translation and adaptation or otherwise.

Open access This is an open access article distributed in accordance with the Creative Commons Attribution Non Commercial (CC BY-NC 4.0) license, which permits others to distribute, remix, adapt, build upon this work non-commercially, and license their derivative works on different terms, provided the original work is properly cited, appropriate credit is given, any changes made indicated, and the use is non-commercial. See: http://creativecommons.org/licenses/by-nc/4.0/.

\section{ORCID iD}

Larisa G Tereshchenko http://orcid.org/0000-0002-6976-1313

\section{REFERENCES}

1 Virani SS, Alonso A, Benjamin EJ, et al. Heart disease and stroke Statistics-2020 update: a report from the American heart association. Circulation 2020;141:e139-596.

2 Koton S, Sang Y, Schneider ALC. Trends in stroke incidence rates in older us adults: an update from the Atherosclerosis risk in communities (ARIC) cohort study. JAMA Neuro/2019.
3 GBD 2016 Lifetime Risk of Stroke Collaborators, Feigin VL, Nguyen $\mathrm{G}$, et al. Global, regional, and country-specific lifetime risks of stroke, 1990 and 2016. N Engl J Med 2018;379:2429-37.

4 Feigin VL, Roth GA, Naghavi M, et al. Global burden of stroke and risk factors in 188 countries, during 1990-2013: a systematic analysis for the global burden of disease study 2013. Lancet Neurol 2016;15:913-24.

5 Malik R, Chauhan G, Traylor M, et al. Multiancestry genome-wide association study of 520,000 subjects identifies 32 loci associated with stroke and stroke subtypes. Nat Genet 2018;50:524-37.

6 Maheshwari A, Norby FL, Roetker NS. Refining prediction of atrial Fibrillation-Related stroke using the P2-CHA2DS2-VASc score. Circulation 2019;139:180-91.

7 Koene RJ, Alraies MC, Norby FL, et al. Relation of the $\mathrm{CHA}_{2} \mathrm{DS}_{2}-$ VASc Score to Risk of Thrombotic and Embolic Stroke in Community-Dwelling Individuals Without Atrial Fibrillation (From The Atherosclerosis Risk in Communities [ARIC] Study). Am J Cardiol 2019;123:402-8.

8 Pirinen J, Eranti A, Knekt P, et al. ECG markers associated with ischemic stroke at young age - a case-control study. Ann Med 2017;49:562-8

9 Ofoma U, He F, Shaffer ML, et al. Premature cardiac contractions and risk of incident ischemic stroke. J Am Heart Assoc 2012;1:e002519.

10 Agarwal SK, Heiss G, Rautaharju PM, et al. Premature ventricular complexes and the risk of incident stroke: the Atherosclerosis risk in communities (ARIC) study. Stroke 2010;41:588-93.

11 Agarwal SK, Chao J, Peace F, et al. Premature ventricular complexes on screening electrocardiogram and risk of ischemic stroke. Stroke 2015;46:1365-7.

12 Tiffany Win T, Ambale Venkatesh B, Volpe GJ, et al. Associations of electrocardiographic $\mathrm{P}$-wave characteristics with left atrial function, and diffuse left ventricular fibrosis defined by cardiac magnetic resonance: the PRIMERI study. Heart Rhythm 2015;12:155-62.

13 Waks JW, Sitlani CM, Soliman EZ, et al. Global electric heterogeneity risk score for prediction of sudden cardiac death in the general population: the Atherosclerosis risk in communities (ARIC) and cardiovascular health (CHS) studies. Circulation 2016;133:2222-34.

14 Waks JW, Tereshchenko LG. Global electrical heterogeneity: a review of the spatial ventricular gradient. J Electrocardiol 2016;49:824-30.

15 Tereshchenko LG, Ghanem RN, Abeyratne A, et al. Intracardiac QT integral on far-field ICD electrogram predicts sustained ventricular tachyarrhythmias in ICD patients. Heart Rhythm 2011;8:1889-94.

16 Haq K, Cao J, Tereshchenko LG. Characteristics of cardiac memory in patients with implanted cardioverter defibrillator: the CAMI study. medRxiv 2019.

17 The ARIC Investigators. The Atherosclerosis risk in communities (ARIC) study: design and objectives. Am J Epidemiol 1989;129:687-702.

18 Chambless LE, Shahar E, Sharrett AR, et al. Association of transient ischemic attack/stroke symptoms assessed by standardized questionnaire and algorithm with cerebrovascular risk factors and carotid artery wall thickness. The ARIC study, 1987-1989. Am J Epidemiol 1996;144:857-66.

19 Perez-Alday EA, Bender A, German D, et al. Dynamic predictive accuracy of electrocardiographic biomarkers of sudden cardiac death within a survival framework: the Atherosclerosis risk in communities (ARIC) study. BMC Cardiovasc Disord 2019;19:255.

20 Jensen K, Howell SJ, Phan F, et al. Bringing critical race praxis into the study of electrophysiological substrate of sudden cardiac death: the ARIC study. J Am Heart Assoc 2020;9:e015012.

21 Howell SJ, German D, Bender A. Does sex modify an association of electrophysiological substrate with sudden cardiac death? The Atherosclerosis Risk in Communities (ARIC) Study bioRxiv 2019;674689.

22 Perez-Alday EA, Li-Pershing Y, Bender A, et al. Importance of the heart vector origin point definition for an $E C G$ analysis: the Atherosclerosis risk in communities (ARIC) study. Comput Biol Med 2019;104:127-38.

23 Thomas JA, A Perez-Alday E, Junell A, et al. Vectorcardiogram in athletes: the sun Valley Ski study. Ann Noninvasive Electrocardiol 2019;24:e12614

24 Okin PM, Roman MJ, Devereux RB, et al. Electrocardiographic diagnosis of left ventricular hypertrophy by the time-voltage integral of the QRS complex. J Am Coll Cardiol 1994;23:133-40.

25 Prineas RJ, Crow RS, Zhang Z-M. The Minnesota code manual of electrocardiographic findings : standards and procedures for measurement and classification. 2nd edn. London: Springer, 2010.

26 White $A D$, Folsom AR, Chambless LE, et al. Community surveillance of coronary heart disease in the Atherosclerosis risk in communities (ARIC) study: methods and initial two years' experience. $J$ Clin Epidemiol 1996;49:223-33. 
27 Rosamond WD, Folsom AR, Chambless LE, et al. Stroke incidence and survival among middle-aged adults: 9-year follow-up of the Atherosclerosis risk in communities (ARIC) cohort. Stroke 1999;30:736-43.

28 Alonso A, Agarwal SK, Soliman EZ, et al. Incidence of atrial fibrillation in whites and African-Americans: the Atherosclerosis risk in communities (ARIC) study. Am Heart J 2009;158:111-7.

29 Soliman EZ, Prineas RJ, Case LD, et al. Ethnic distribution of ECG predictors of atrial fibrillation and its impact on understanding the ethnic distribution of ischemic stroke in the Atherosclerosis risk in communities (ARIC) study. Stroke 2009;40:1204-11.

30 Tereshchenko LG, Henrikson CA, Sotoodehnia N, et al. Electrocardiographic deep terminal negativity of the $P$ wave in $\mathrm{V}(1)$ and risk of sudden cardiac death: the Atherosclerosis Risk in Communities (ARIC) study. J Am Heart Assoc 2014;3:e001387.

31 Tereshchenko LG, Shah AJ, Li Y, et al. Electrocardiographic deep terminal negativity of the $\mathrm{P}$ wave in $\mathrm{V} 1$ and risk of mortality: the National health and nutrition examination survey III. J Cardiovasc Electrophysiol 2014;25:1242-8.

32 The ARIC Study Group. High-resolution B-mode ultrasound scanning methods in the Atherosclerosis Risk in Communities Study (ARIC). J Neuroimaging 1991;1:68-73.

33 Ohira T, Shahar E, Chambless LE, et al. Risk factors for ischemic stroke subtypes: the Atherosclerosis Risk in Communities study. Stroke 2006;37:2493-8.

34 Folsom AR, Yatsuya H, Psaty BM, et al. Carotid intima-media thickness, electrocardiographic left ventricular hypertrophy, and incidence of intracerebral hemorrhage. Stroke 2011;42:3075-9.

35 Blackburn H, Keys A, Simonson E, et al. The electrocardiogram in population studies. A classification system. Circulation 1960;21:1160-75.

36 Hicks CW, Yang C, Ndumele CE, et al. Associations of obesity with incident hospitalization related to peripheral artery disease and critical limb ischemia in the ARIC study. J Am Heart Assoc 2018;7:e0 08644-e44

37 Eriksson H, Caidahl K, Larsson B, et al. Cardiac and pulmonary causes of dyspnoea-validation of a scoring test for clinicalepidemiological use: the Study of Men Born in 1913. Eur Heart $J$ 1987;8:1007-14.

38 Matsushita K, Selvin E, Bash LD, et al. Risk implications of the new CKD epidemiology collaboration (CKD-EPI) equation compared with the MDRD study equation for estimated GFR: the Atherosclerosis risk in communities (ARIC) study. Am J Kidney Dis 2010;55:648-59.

39 Richardson MT, Ainsworth BE, Wu HC, et al. Ability of the Atherosclerosis Risk in Communities (ARIC)/Baecke Questionnaire to assess leisure-time physical activity. Int J Epidemiol 1995;24:685-93.

40 Cox NJ. Speaking Stata: in praise of trigonometric predictors. Stata J 2006;6:561-79.

41 O'Neal WT, Kamel H, Zhang Z-M, et al. Advanced interatrial block and ischemic stroke: the Atherosclerosis risk in Communities study. Neurology 2016;87:352-6.

42 von Elm E, Altman DG, Egger M, et al. The strengthening the reporting of observational studies in epidemiology (STROBE) statement: guidelines for reporting observational studies. Ann Intern Med 2007;147:573-7.

43 Tereshchenko LG, Henrikson CA, Cigarroa J, et al. Comparative effectiveness of interventions for stroke prevention in atrial fibrillation: a network meta-analysis. J Am Heart Assoc 2016;5 doi:10.1161/ JAHA.116.003206

44 Watson T, Shantsila E, Lip GYH. Mechanisms of thrombogenesis in atrial fibrillation: Virchow's triad revisited. Lancet 2009;373:155-66.

45 Brambatti M, Connolly SJ, Gold MR, et al. Temporal relationship between subclinical atrial fibrillation and embolic events. Circulation 2014;129:2094-9.

46 Camen S, Ojeda FM, Niiranen T, et al. Temporal relations between atrial fibrillation and ischaemic stroke and their prognostic impact on mortality. Europace 2020;22:522-9.

47 Calenda BW, Fuster V, Halperin JL, et al. Stroke risk assessment in atrial fibrillation: risk factors and markers of atrial myopathy. Nat Rev Cardiol 2016;13:549-59.

48 German DM, Kabir MM, Dewland TA, et al. Atrial fibrillation predictors: importance of the electrocardiogram. Ann Noninvasive Electrocardiol 2016;21:20-9.

49 Ganesan AN, Chew DP, Hartshorne T, et al. The impact of atria fibrillation type on the risk of thromboembolism, mortality, and bleeding: a systematic review and meta-analysis. Eur Heart $J$ 2016;37:1591-602

50 Vanassche T, Lauw MN, Eikelboom JW, et al. Risk of ischaemic stroke according to pattern of atrial fibrillation: analysis of 6563 aspirin-treated patients in ACTIVE-A and AVERROES. Eur Heart $J$ 2015;36:281-7.

51 Boriani G, Glotzer TV, Santini M, et al. Device-detected atrial fibrillation and risk for stroke: an analysis of $>10,000$ patients from the SOS AF project (Stroke preventiOn Strategies based on Atrial Fibrillation information from implanted devices). Eur Heart $J$ 2014:35:508-16.

52 Turakhia MP, Ziegler PD, Schmitt SK, et al. Atrial fibrillation burden and short-term risk of stroke: case-crossover analysis of continuously recorded heart rhythm from cardiac electronic implanted devices. Circ Arrhythm Electrophysiol 2015;8:1040-7.

53 Schnabel RB, Haeusler KG, Healey JS, et al. Searching for atrial fibrillation poststroke: a white paper of the AF-SCREEN international collaboration. Circulation 2019;140:1834-50.

54 Murthy S, Rizzi P, Mewton N, et al. Number of P-wave fragmentations on P-SAECG correlates with infiltrated atrial fat. Ann Noninvasive Electrocardiol 2014;19:114-21.

55 Tereshchenko LG, Rizzi P, Mewton N, et al. Infiltrated atrial fat characterizes underlying atrial fibrillation substrate in patients at risk as defined by the ARIC atrial fibrillation risk score. Int J Cardiol 2014;172:196-201.

56 Shvilkin A, Bojovic B, Vajdic B, et al. Vectorcardiographic determinants of cardiac memory during normal ventricular activation and continuous ventricular pacing. Heart Rhythm 2009;6:943-8.

57 Shvilkin A, Huang HD, Josephson ME. Cardiac memory: diagnostic tool in the making. Circ Arrhythm Electrophysiol 2015;8:475-82.

58 Sakamoto $\mathrm{Y}$, Inden $\mathrm{Y}$, Okamoto $\mathrm{H}$, et al. T-wave changes of cardiac memory caused by frequent premature ventricular contractions originating from the right ventricular outflow tract. J Cardiovasc Electrophysiol 2019;30:1549-56.

59 Josephson ME, Callans DJ. Using the twelve-lead electrocardiogram to localize the site of origin of ventricular tachycardia. Heart Rhythm 2005;2:443-6.

60 de Vries LJ, Hendriks AA, Yap SC, et al. Procedural and long-term outcome after catheter ablation of idiopathic outflow tract ventricular arrhythmias: comparing manual, contact force, and magnetic navigated ablation. Europace 2018;20:ii22-7.

61 Seibolt L, Maestas C, Lazkani M, et al. Rate-related left bundle branch block and cardiac memory in a patient with bradycardia: case report and literature review. Clin Cardiol 2018;41:1097-102.

62 Akoudad S, Portegies MLP, Koudstaal PJ, et al. Cerebral microbleeds are associated with an increased risk of stroke: the Rotterdam study. Circulation 2015;132:509-16.

63 Yamada M. Cerebral amyloid angiopathy: emerging concepts. J Stroke 2015;17:17-30.

64 Graff-Radford J, Botha H, Rabinstein AA, et al. Cerebral microbleeds: prevalence and relationship to amyloid burden. Neurology 2019;92:e253-62.

65 Sekijima Y, Yazaki M, Oguchi K, et al. Cerebral amyloid angiopathy in posttransplant patients with hereditary ATTR amyloidosis. Neurology 2016;87:773-81.

66 Alexander KM, Orav J, Singh A, et al. Geographic disparities in reported us amyloidosis mortality from 1979 to 2015: potential Underdetection of cardiac amyloidosis. JAMA Cardiol 2018;3:865-70.

67 Tanskanen M, Peuralinna T, Polvikoski T, et al. Senile systemic amyloidosis affects $25 \%$ of the very aged and associates with genetic variation in alpha2-macroglobulin and tau: a populationbased autopsy study. Ann Med 2008;40:232-9.

68 González-López E, Gallego-Delgado M, Guzzo-Merello G, et al. Wild-type transthyretin amyloidosis as a cause of heart failure with preserved ejection fraction. Eur Heart J 2015;36:2585-94.

69 Gillmore JD, Maurer MS, Falk RH, et al. Nonbiopsy diagnosis of cardiac transthyretin amyloidosis. Circulation 2016;133:2404-12.

70 Alexander KM, Singh A, Falk RH. Novel pharmacotherapies for cardiac amyloidosis. Pharmacol Ther 2017;180:129-38.

71 Cyrille NB, Goldsmith J, Alvarez J, et al. Prevalence and prognostic significance of low QRS voltage among the three main types of cardiac amyloidosis. Am J Cardiol 2014;114:1089-93.

72 Giancaterino S, Urey MA, Darden D, et al. Management of Arrhythmias in Cardiac Amyloidosis. JACC Clin Electrophysiol 2020;6:351-61.

73 Kaiser J, Schebesch K-M, Brawanski A, et al. Long-term follow-up of cerebral amyloid Angiopathy-Associated intracranial hemorrhage reveals a high prevalence of atrial fibrillation. J Stroke Cerebrovasc Dis 2019;28:104342.

74 Charidimou A, Shoamanesh A, Al-Shahi Salman R, et al. Cerebral amyloid angiopathy, cerebral microbleeds and implications for anticoagulation decisions: the need for a balanced approach. Int J Stroke 2018;13:117-20. 